\title{
Opioid Craving in Human Laboratory Settings: a Review of the Challenges and Limitations
}

\author{
Kimberly Goodyear ${ }^{1,2,3}$ (D) Carolina L. Haass-Koffler ${ }^{1,2,3}$ \\ Published online: 24 October 2019 \\ (C) The American Society for Experimental NeuroTherapeutics, Inc. 2019
}

\begin{abstract}
There are many factors that need to be taken into consideration when measuring craving in a human laboratory study. This review summarizes and discusses some of the major challenges researchers are faced with when assessing opioid craving in clinical research. First, there are wide range of available assessments that have been developed for measuring craving and depending on the research questions or the underlying constructs targeted, some may be more appropriate than others. In addition to establishing the methodological point of departure for designing a study with craving, there are also different participant conditions and characteristics that need to be evaluated when selecting among the large selection of assessments available. Participant conditions/characteristics can influence the degree or frequency of opioid craving experienced. Lastly, there can be contextual conditions that affect opioid cravings such as a stressful environment that may alter cue saliency. It is recommended that researchers carefully consider the different constituents that contribute to opioid craving and to ensure a comprehensive evaluation when assessing each participant. A more thorough consideration of these challenges can help us to understand the optimal ways to measure one important and complex component of addiction.
\end{abstract}

Key·Words measurement $\cdot$ craving $\cdot$ human laboratory $\cdot$ assessments $\cdot$ opioids

\section{Introduction}

The role of craving in drug-seeking behaviors has been wellestablished in addiction research over the past 60 years. The contribution of craving in addictions was further substantiated after being clinically recognized in the latest edition of the Diagnostic and Statistical Manual of Mental Disorders (DSM5) as diagnostic criteria for substance use disorders [7]. Despite

Electronic supplementary material The online version of this article (https://doi.org/10.1007/s13311-019-00791-8) contains supplementary material, which is available to authorized users.

Kimberly Goodyear

kimberly_goodyear@brown.edu

1 Center for Alcohol and Addiction Studies, Department of Behavioral and Social Sciences, Brown University, Providence, RI, USA

2 Section on Clinical Psychoneuroendocrinology and Neuropsychopharmacology, National Institute on Alcohol Abuse and Alcoholism; and National Institute on Drug Abuse, National Institutes of Health, Bethesda, MD, USA

3 Center for Alcohol and Addiction Studies, Department of Psychiatry and Human Behavior, Brown University, Providence, RI, USA the clinical recognition and the large volume of research on craving, the measurement of craving in human laboratory studies has been a source of debate among researchers [13]. The challenges with measuring craving include the definition of craving [25], the clinical utility of craving and the reliability of subjective craving responses for predicting future behaviors [28]. Furthermore, craving can be broken down to tonic (i.e., longer-term) and phasic (i.e., in the moment) cravings that may be influenced by different conditions [10, 27]. For instance, participant characteristics or conditions may change tonic craving, while contextual conditions such as cue-exposure may influence phasic craving.

In addition to measuring general craving in addictive behaviors, another complicating factor is assessing craving specifically for opioids. Non-medical opioid use continues to rise in the United States and the research on opioid craving has also grown exponentially. Despite the increase in craving research, the measurement of opioid craving continues to present some issues. This review discusses three important methodological challenges for measuring opioid craving in laboratory settings: (1) how opioid craving is assessed, (2) the spectrum of the study participant conditions and characteristics (e.g., withdrawal syndrome), and (3) the contextual (e.g., naturalistic environment) conditions that may influence opioid craving in the laboratory. 


\section{Opioid Craving Assessment}

The first methodological challenge is deciding how to measure craving in the laboratory. Craving is generally assessed by participants' subjective responses to scales or questionnaires. A visual analog scale (VAS) is a common instantmeasurement instrument used to assess how strong an urge or desire is across a continuum. The values on the VAS typically range from different point values (e.g., 1-10, 1-100) with a higher value indicating a stronger desire or urge to use opioids. The VAS can be easily adapted to different paradigms and research questions, and therefore, no standardized verbiage has been established. One disadvantage of singleitem measures, such as the VAS, is that the reliability (internal consistency) cannot be measured. However, a considerable advantage of using a VAS is that it can be utilized as a continuous variable rather than a binary or discrete response. This option captures the extent of craving, which more accurately reflects the spectrum of the disorder as described in the DSM5. It also allows a variety of analysis options that can offer insight into sources of variation and can accommodate smaller samples, which is common in human laboratory studies.

While unidimensional assessments are easy to implement, multi-item questionnaires allow for a multidimensional measurement of craving across different levels [30]. Several multi-item questionnaires have been developed to measure opioid craving. The Desires for Drug Questionnaire (DDQ) and Obsessive Compulsive Drug Use Scale (OCDUS) [8], the Heroin Craving Questionnaire (HCQ) [29] and the Opioid Craving Scale (OCS) [31] were derived from alcohol and cocaine craving questionnaires. The DDQ, OCDUS, and HCQ were initially validated for heroin craving and have since been adapted for other opioids. The multi-item questionnaires are not necessarily comparable across measures. For instance, some measure instant craving in the moment (right now) and others measure general craving (typically within the past week) that can be retrospective. The OCDUS and HCQ measure more general craving, the DDQ measures instant craving, and the OCS measures instant craving and general craving. General craving assessments may be more suitable for longitudinal studies or techniques that do not need to be done retrospectively (e.g., ecological momentary assessment). Furthermore, multi-item questionnaires can measure different underlying dimensions or constructs of craving. For instance, the OCDUS consists of heroin thoughts and interference, desire and control, and resistance to thoughts and intentions factors, while the DDQ consists of desire and intention, negative reinforcement, and control factors [8]. The components of craving are not definitive and depending on the circumstances, one questionnaire may be more appropriate for capturing craving experienced by a participant.

\section{Participant Conditions and Characteristics}

Different participant conditions and characteristics need to be taken into account when measuring craving, as the degree of craving experienced at any time can vary according to a participant's status (e.g., in recovery, treatment seeking, currently using), individual characteristics, or the type of opioids used. In recent years, more attention on participants' statuses and individual differences have come to light in alcohol research $[12,13]$. For instance, a study showed that treatment-seeking individuals with alcohol use disorder (AUD) had higher alcohol craving compared to non-treatment-seeking individuals [22]. Although not directly measured, it could be surmised that craving for other types of substances such as opioids, could also vary as a function of treatment-seeking status. Due to ethical issues and feasibility of conducting a study, researchers may be limited by the population that they can recruit (treatment versus non-treatment seekers).

When designing a human laboratory study with craving the general nomenclature used when describing opioids needs to be accounted for. Specific substances such as heroin, painkillers, fentanyl, etc. are classified under the umbrella opioid term, however, "opioids" can mean something different to each person. A study comparing individuals who use prescription opioids to heroin demonstrated that craving responses to drug paraphernalia were moderated by the opioid type [21]. Therefore, assessments for general opioid craving may not be fully applicable when there is specificity of the type of opioid used. In addition, there has been a lack of research on fentanyl craving in general, and as the use of illicit fentanyl and other synthetic opioids are on the rise [26], there needs to be greater focus on the different types of opioid craving.

Individuals who use opioids also commonly use other substances. Someone using different substances (e.g., heroin and painkillers) may have competing urges and/or their cravings could overlap. A craving for one type of opioid may be stronger or it could potentially negate another craving. Assessing all types of opioid cravings for multi-substance users would increase our understanding about overlapping or interacting processes that may exist between different substances.

Withdrawal syndrome is another example of a personspecific condition or trigger. When someone is undergoing withdrawal, they have a physical need to obtain and take a drug. The constellation of symptoms in the withdrawal experience may also influence the desire to use a substance. There may be an increase in cravings that occur when someone has the urge to use a substance and the physiological need to alleviate the withdrawal symptoms overlap. For instance, there have been several studies in the AUD field that have compared treatment-seekers' characteristics from the national multi-site clinical trial COMBINE study [2], with nontreatment seekers' characteristics conducted in California [22] and Rhode Island (Haass-Koffler et al., under review). 
Both works reported that treatment-seekers, being more likely to be abstinent (more abstinent days), present higher level of withdrawal symptoms than non-treatment seekers.

Another example is someone taking medication such as methadone or buprenorphine to treat their opioid use. People who are on a stable dose of an opioid replacement therapy (ORT) may experience less or have fluctuating urges compared to people not on medication. Their urges may be contingent on the amount and stability of their medication dose. The type of medication (e.g., methadone versus buprenorphine) may also vary the amount of craving an individual experiences [20]. This speaks to the need for utilizing structured craving biobehavioral assays to provide support in the assessment of pharmacotherapies outcomes, not only in the research and discovery setting, but also to confirm craving differences among types of medication in clinical practice. Further, the efficacy of the medication may also be dependent on whether or not someone is receiving medication-assisted treatment (MAT) [6] and that in turn, can also change a craving response.

Another person-specific condition is response to pain. A person with chronic pain may have a physical need to treat their pain with opioids and that could potentially change the type or the degree of craving they have. However, pain is not necessarily separate from addiction and individuals who have chronic pain may experience craving for opioids in the same way as someone without chronic pain [24]. In addition, a study illustrated that abstinent individuals with heroin dependence had increased sensitivity to pain and pain distress positively correlated with cue-induced heroin craving [23]. This study further highlights the complexity of person-specific factors such as response to pain and how that can affect opioid craving.

In addition to individual conditions, there may also be participant characteristics that may influence craving. For instance, a study investigating the role of genetics in alcohol craving demonstrated that among individuals with alcohol dependence, there may be genetic factors affecting alcohol craving [1]. Furthermore, a national multisite trial demonstrated that women had higher craving for opioids compared to men [3], which highlights gender differences. Greater emphasis on clinical profiles, especially in regard to gender, should be considered when determining opioid craving.

\section{Contextual Conditions that May Influence Cravings}

Lastly, there may be contextual conditions that increase a craving response across individuals. Commonly, craving is measured through cue elicitation. Cue-reactivity is a controlled laboratory paradigm in which an individual's behavioral, biological, physiological, and subjective responses to specific drug-related cues can be measured. Cues can be in the form of photos, videos, scripts, or handling the actual drug or other paraphernalia (e.g., a sealed bottle of real opioid prescription pills, spoons, pill crushers, straws, small square pieces of aluminum foil, money) [4]. However, when dealing with illicit drugs like heroin, it can be difficult, or not even feasible to include the actual drug in a cue-reactivity paradigm. Sometimes there may even be precursors to the cuereactivity paradigm that will increase craving. Examples are priming doses of the drug or a stress induction, which can be done with behavioral stressors (e.g., Trier Social Stress Test (TSST)) [16], physiological stressors (e.g., drugs like yohimbine) [11] and imagery exposure (e.g. stressful or drug-cue personal experiences) [15]. Depending on the precursor, craving may vary as a function of the environmental setting or across participants. Some individuals may be triggered by an initial dose of their preferred drug while others may not feel a comparable urge. Along the same lines, the extent that an individual feels stressed may differ for each participant. In addition to stress conditions, subjective craving can also be measured in conjunction with modalities such as cardiovascular measures (e.g., heart rate and blood pressure). For instance, a study examining craving, cardiovascular responses to stress, and drug cues in recently abstinent individuals with opioid use disorder treated with naltrexone showed that craving increased in both cue conditions and cardiovascular responses only increased during the stress condition [15]. These findings highlight the array of conditions and arousal responses that can be assessed during cue-induced craving. Furthermore, the methodological issues experienced in behavioral laboratories can also translate to functional magnetic resonance imaging (fMRI) paradigms as the same concerns can arise when measuring craving during a scan. Neuroimaging researchers face even more obstacles, as creating a naturalistic and multisensory environment to induce craving during an fMRI scan can be a difficult endeavor [9]. Craving measured during an fMRI scan typically needs to be done iteratively and this can be burdensome to some participants and can also reduce external validity. Nonetheless, fMRI elaborates on self-report assessments by showing the involvement of different brain regions during craving. This provides researchers with more substantive information about the many components that craving is comprised of.

Furthermore, there may be environmental triggers that change an individual's degree of craving. For instance, risk of relapse may be potentially higher in someone who is more sensitive to environmental triggers such as a stressful or unfamiliar environment. Depending on the time and place that craving is assessed, there may not be an accurate depiction of how someone typically experiences craving. Assessing craving in a laboratory may in itself alter a craving response without any sort of induction or paradigm. Therefore, there may be environmental limitations of assessing craving in a 
laboratory that are not seen in real-world settings. However, in more recent years, the creation of more naturalistic environments to increase cue saliency for participants has gained momentum in alcohol studies. For example, to mimic real-life conditions, researchers have constructed bar-like laboratories $[14,19]$, included confederates in studies to stimulate real-life interactions with peers [18] and created virtual reality environments [5] to assess alcohol craving. In comparison to alcohol studies, creating naturalistic environments to assess opioid craving remains a challenge. Since alcohol is a legal substance and many opioids are not, recreating a naturalistic environment (e.g. bar-laboratory) to elicit alcohol craving is more feasible. In addition, there is a lack of understanding about the dynamics of group contexts, as opioids used in group settings are done so illicitly. One study showed that participants misused opioids primarily in households [17]; however, there are limited studies on location or environmental contexts for opioid use. Researchers are limited in the ways to recreate naturalistic environments for participants who use opioids.

\section{Conclusions}

There is a multitude of factors that need to be considered when designing a human laboratory study with opioid craving. Among some of the factors are how craving is assessed, participant conditions and characteristics, and the contextual conditions that may influence craving. As research on opioid craving continues to grow, ensuring the proper measurement can present methodological challenges. When designing human laboratory studies, behaviors need to be quantifiable in some way, but the impetus that triggers craving may not necessarily be something that is measurable. Given that possible limitation, craving measures should be refined in such a way that they can fully capture what is intended to measure. While the focus of this review was on the challenges and limitations of designing a human laboratory study to assess opioid craving, it is important to mention that there are several advantages to assessing craving in the human laboratory in addition to measuring craving in the naturalistic environment. Laboratory settings for evaluating opioid craving allow for controlled environments without the introduction of additional variables, reduction of the knowledge gap between preclinical studies and randomized clinical trials, well-controlled experimental manipulations such as stress reduction and a safe and private environment for participants. Moreover, some of the recommendations can be applied to other types of studies (e.g., ambulatory studies). In the same way that human laboratory researchers should refine the way they define participant conditions and characteristics and contextual conditions, researchers designing ambulatory studies can also fully detail how they capture the different aspects of opioid craving.
Moving forward, a "wide-lens" approach should be employed such that different contextual and person factors are considered when designing studies to assess opioid craving. The following recommendations are suggested. Researchers should be aware that there are many different assessments available and they should be selective in choosing the appropriate craving assessment. Participant conditions or characteristics should be controlled for or noted when analyzing results and discussing findings. For instance, participants who are treatment-seeking and on opioid replacement therapy would have a reduction in craving compared to nontreatment-seeking individuals. Therefore, it is important to note these differences when discussing the implications of the research and to also include covariates during analysis to control for those differences if possible. The same approach for assessing participant conditions or characteristics should be taken with contextual conditions. When choosing contextual conditions that may influence craving, researchers should be mindful in the way they assess and approach craving and how they interpret their results and findings. More attention is being put forth to opioid craving and this will help to increase our understanding of such a complex psychological construct, which can serve to improve the ways in which we assess addictive behaviors in the laboratory.

Acknowledgments Dr. Goodyear is supported by the National Institute on Alcohol Abuse and Alcoholism (K01 AA026874) and previously by the 5T32AA007459 training grant. Dr. Haass-Koffler is supported by the National Institute on Alcohol Abuse and Alcoholism (K01 AA023867; R01 AA026589; R01 AA027760) and by the National Institute of General Medical Sciences (NIGMS), Center of Biomedical Research Excellence (COBRE, P20 GM130414).

Required Author Forms Disclosure forms provided by the authors are available with the online version of this article.

\section{Compliance with Ethical Standards}

Conflict of Interest The authors declare that they have no conflict of interest.

\section{References}

1. Agrawal A, Wetherill L, Bucholz KK, et al. Genetic influences on craving for alcohol. Addict Behav. 2013;38(2):1501-8. https://doi. org/10.1016/j.addbeh.2012.03.021.

2. Anton RF, O'Malley SS, Ciraulo DA, et al. Combined pharmacotherapies and behavioral interventions for alcohol dependence: the COMBINE study: a randomized controlled trial. Jama. 2006;295(17):2003-17. https://doi.org/10.1001/jama.295.17.2003.

3. Back SE, Payne RL, Wahlquist AH, et al. Comparative profiles of men and women with opioid dependence: results from a national multisite effectiveness trial. Am J Drug Alcohol Abuse. 2011;37(5): 313-23. https://doi.org/10.3109/00952990.2011.596982.

4. Back SE, Gros DF, Price M, et al. Laboratory-induced stress and craving among individuals with prescription opioid dependence. 
Drug Alcohol Depend. 2015;155:60-7. https://doi.org/10.1016/j. drugalcdep.2015.08.019.

5. Bordnick PS, Traylor A, Copp HL, et al. Assessing reactivity to virtual reality alcohol based cues. Addict Behav. 2008;33(6):74356. https://doi.org/10.1016/j.addbeh.2007.12.010.

6. Connery HS. Medication-assisted treatment of opioid use disorder: review of the evidence and future directions. Harvard Rev Psychiatry. 2015;23(2):63-75. https://doi.org/10.1097/hrp. 0000000000000075.

7. First MB. Structured Clinical Interview for the DSM (SCID). In The Encyclopedia of Clinical Psychology: Wiley. 2014.

8. Franken IHA, Hendriks VM, van den Brink W. Initial validation of two opiate craving questionnaires: The Obsessive Compulsive Drug Use Scale and the Desires for Drug Questionnaire. Addict Behav. 2002;27(5):675-85. https://doi.org/10.1016/S03064603(01)00201-5.

9. Goodyear K. Multisensory Environments to Measure Craving During Functional Magnetic Resonance Imaging. Alcohol and Alcoholism. 2019;54(3), 193-195.

10. Grace AA. The tonic/phasic model of dopamine system regulation and its implications for understanding alcohol and psychostimulant craving. Addiction. 2000;95(Suppl 2):S119-28.

11. Greenwald MK, Lundahl LH, Steinmiller CL. Yohimbine increases opioid-seeking behavior in heroin-dependent, buprenorphinemaintained individuals. Psychopharmacology (Berl). 2013;225(4): 811-24. https://doi.org/10.1007/s00213-012-2868-9.

12. Haass-Koffler CL, Kenna GA. Bacchus by Caravaggio as the Visual Diagnosis of Alcohol Use Disorder from the Fifth Edition of the Diagnostic and Statistical Manual of Mental Disorders (DSM-5). Front Psychiatry. 2013;4:86. https://doi.org/10.3389/ fpsyt.2013.00086.

13. Haass-Koffler CL, Leggio L, Kenna GA. Pharmacological Approaches to Reducing Craving in Patients with Alcohol Use Disorders. CNS Drugs. 2014;28(4):343-60. https://doi.org/10. 1007/s40263-014-0149-3.

14. Haass-Koffler CL, Goodyear K, Zywiak WH, Leggio L, Kenna GA, Swift RM. Comparing and Combining Topiramate and Aripiprazole on Alcohol-Related Outcomes in a Human Laboratory Study. Alcohol Alcohol. 2018;53(3):268-76. https:// doi.org/10.1093/alcalc/agx108.

15. Hyman SM, Fox H, Hong K-IA, Doebrick C, Sinha R. Stress and drug-cue-induced craving in opioid-dependent individuals in naltrexone treatment. Exp Clin Psychopharmacol. 2007;15(2):134-43. https://doi.org/10.1037/1064-1297.15.2.134.

16. Kirschbaum C, Pirke K-M, Hellhammer DH. The 'Trier Social Stress Test' $-\mathrm{a}$ tool for investigating psychobiological stress responses in a laboratory setting. Neuropsychobiology. 1993;28(12):76-81.

17. Lankenau SE, Teti M, Silva K, Bloom JJ, Harocopos A, Treese M. Initiation into prescription opioid misuse amongst young injection drug users. Int J Drug Policy. 2012;23(1):37-44. https://doi.org/10. 1016/j.drugpo.2011.05.014

18. Larsen H, Engels RCME, Granic I, Overbeek G. An experimental study on imitation of alcohol consumption in same-sex dyads.
Alcohol and Alcoholism. 2009;44(3):250-5. https://doi.org/10. 1093/alcalc/agp002.

19. Leggio L, Zywiak WH, Edwards SM, Tidey JW, Swift RM, Kenna GA. A preliminary double-blind, placebo-controlled randomized study of baclofen effects in alcoholic smokers. Psychopharmacology. 2015;232(1):233-43. https://doi.org/10. 1007/s00213-014-3652-9.

20. Ling W, Wesson DR. Clinical efficacy of buprenorphine: comparisons to methadone and placebo. Drug Alcohol Depend. 2003;70(2, Supplement):S49-57. https://doi.org/10.1016/S0376-8716(03) 00059-0.

21. McHugh RK, Fulciniti F, Mashhoon Y, Weiss RD. Cue-induced craving to paraphernalia and drug images in opioid dependence. Am J Addict. 2016;25(2):105-9. https://doi.org/10.1111/ajad. 12344

22. Ray LA, Bujarski S, Yardley MM, Roche DJO, Hartwell EE. Differences between treatment-seeking and non-treatment-seeking participants in medication studies for alcoholism: do they matter? Am J Drug Alcohol Abuse. 2017;43(6):703-10. https://doi.org/10. 1080/00952990.2017.1312423.

23. Ren Z-Y, Shi J, Epstein DH, Wang J, Lu L. Abnormal pain response in pain-sensitive opiate addicts after prolonged abstinence predicts increased drug craving. Psychopharmacology. 2009;204(3):423-9. https://doi.org/10.1007/s00213-009-1472-0.

24. Rosenblum A, Marsch LA, Joseph H, Portenoy RK. Opioids and the treatment of chronic pain: controversies, current status, and future directions. Exp Clin Psychopharmacol. 2008;16(5):405-16. https://doi.org/10.1037/a0013628.

25. Sayette MA, Shiffman S, Tiffany ST, Niaura RS, Martin CS, Schadel WG. The measurement of drug craving. Addiction. 2000;95(8s2):189-210.

26. Schuchat A, Houry D, Guy GP Jr. New Data on Opioid Use and Prescribing in the United StatesNew Data on Opioid Use and Prescribing, 2006-2015New Data on Opioid Use and Prescribing, 2006-2015. Jama. 2017;318(5):425-6. https://doi.org/10.1001/ jama.2017.8913.

27. Shiffman S. Comments on craving. Addiction. 2000;95(8s2):1715 .

28. Tiffany ST, Wray JM. The clinical significance of drug craving. Ann N Y Acad Sci. 2012;1248(1):1-17.

29. Tiffany ST, Singleton E, Haertzen CA, Henningfield JE. The Development of a Heroin Craving Questionnaire. In: [unpublished manuscript] Purdue University. 1993.

30. Tiffany ST, Carter BL, Singleton EG. Challenges in the manipulation, assessment and interpretation of craving relevant variables. Addiction. 2000;95(Suppl 2):S177-87.

31. Weiss RD, Griffin ML, Hufford C. Craving in hospitalized cocaine abusers as a predictor of outcome. Am J Drug Alcohol Abuse. 1995;21(3):289-301.

Publisher's Note Springer Nature remains neutral with regard to jurisdictional claims in published maps and institutional affiliations. 